\title{
Educação em enfermagem e os desafios para a promoção de saúde*
}

\author{
Nursing education and the challenges for health promotion \\ Educación en enfermería y los desafíos para la promoción de salud
}

\begin{abstract}
Kênia Lara da Silva', Roseni Rosângela de Sena', Maria Jose Cabral Grillo', Natália de Cássia Horta', Priscilla Malta Coelho Prado'

'Universidade Federal de Minas Gerais. Departamento de Enfermagem.

Núcleo de Estudos e Pesquisas sobre Ensino e Prática de Enfermagem, Belo Horizonte, MG
\end{abstract}

Submissão: $31 / 03 / 2008$

Aprovação: 09/12/2008

\begin{abstract}
RESUMO
Estudo descritivo-exploratório de abordagem Qualitativa cujo objetivo foi analisar a abordagem da promoção da saúde na formação do enfermeiro. Os sujeitos foram docentes, estudantes e profissionais Que recebem os estudantes nos cenários de aprendizagem de dois cursos de graduação em enfermagem de Minas Gerais/Brasil. Há indicativos da incorporação da promoção da saúde como referencial para a formação do enfermeiro favorecidas pela articulação ensino-serviço como importante estratégia neste processo. Entretanto, carecem de maior valorização como condição para sustentabilidade ao processo de mudança na formação do enfermeiro. Conclui-se pela necessidade de ações estratégicas para a modificação das práticas sanitárias e de ensino potencializando as "boas" práticas de promoção da saúde na superação dos desafios para as mudanças na formação do enfermeiro.
\end{abstract}

Descritores: Educação em enfermagem; Promoção da saúde; Integração docente assistencial.

\section{ABSTRACT}

This is a descriptive-exploratory study with Qualitative approach whose objective was analyze the health promotion on nursing education. The subjects were teachers, students \& professionals whom receive the students on the scenarios of learningship of two courses of graduation in Minas Gerais/Brazil. The results indicate the incorporation of health promotion as a referencing for nursing formation favored by school-service articulation as well as an important strategy. In the mean time, it needs major valorization as a condition to maintain the changes occurred in nursing formation. It concludes that is necessary strategic actions for change the sanitary and education practices involving the "good" practices of health promotion on overcoming the challenges to change the nursing education.

Descriptors: Education, Nursing; Health Promotiion; Teaching care integration services.

\section{RESUMEN}

Se trata de un estudio descriptivo exploratorio, con enfoque cualitativo, cuyo objetivo fue analizar cómo se trata la promoción de la salud en la formación del enfermero. Los sujetos fueron docentes, estudiantes y profesionales de los alumnos en los escenarios de aprendizaje de dos cursos de graduación en enfermería de Minas Gerais/Brasil. Hay indicativos de la incorporación de la promoción de la salud como referente para la formación del enfermero favorecida por la articulación enseñanza-trabajo como importante estrategia en este proceso. Sin embargo, precisa ser más valorada como condición para sustentar el proceso de cambio en la formación del enfermero. Como conclusión, podemos afirmar Que se necesitan acciones estratégicas para modificar las prácticas sanitarias y de enseñanza así como también potenciar las "buenas" prácticas de promoción de la salud en la superación de los desafíos para los cambios en la formación del enfermero.

Descriptores: Educación en Enfermería; Promoción de la salud; Servicios de integración docente asistencial.

*Trabalho apresentado ao $59^{\circ}$ Congresso Brasileiro de Enfermagem/Brasília, em dezembro de 2007. Classificado em Io lugar no Prêmio Vilma de Carvalho. Pesquisa financiada pela Fundação de Amparo à Pesquisa do Estado de Minas Gerais (FAPEMIG), sob processo número II 560 FAPEMIG/EENF/EMI/SUS TECNOASSISTENCIAL.

Correspondência: Kênia Lara da Silva. Rua Cornélio Cerqueira, 855/20I, Pe. Eustáquio. CEP 30730-530. Belo Horizonte, MG. 


\section{INTRODUÇÃO}

O paradigma biomédico dominante nas políticas públicas de saúde e de assistência social sustentado em uma concepção de saúde restrita à dimensão biológica e individual mostra-se insuficiente para atender às necessidades e demandas sociais de saúde da população. Consequentemente, passa a haver Questionamentos por diversos setores organizados da sociedade, principalmente a partir da segunda metade da década de setenta, gerando a formulação da proposta de reforma do modelo tecnoassistencial. Os debates realizados na $8^{\mathrm{a}}$ Conferência Nacional de Saúde e, posteriormente, na definição do Sistema único de Saúde e de seus princípios na Constituição da República, em 1988 , são marcos de um movimento Que busca construir um novo modelo de atenção à saúde no Brasil.

Partindo da análise dos processos de trabalho e do conceitochave de organização social da prática médica, tal movimento opera uma leitura socializante da problemática evidenciada pela crise da medicina mercantilizada bem como de sua ineficiência, enQuanto possibilidade de organização de um sistema de saúde capaz de responder as demandas prevalentes, organizado de forma democrática em sua gestão e administrado com base na racionalidade do planejamento ${ }^{(1)}$.

No entanto, como atesta Feuerwerker ${ }^{(2)}$, a prática cotidiana dos serviços de saúde orientados pelo novo paradigma - o SUS real, segundo a própria autora - está muito distante da proposta formulada pelo movimento da Reforma Sanitária, sendo o modelo tecnoassistencial, a organização da gestão e as maneiras como se faz a política de saúde os pontos críticos dessa situação.

Para as pesquisadoras, o projeto em curso no Brasil, Que prioriza a atenção primária, com ênfase na promoção da saúde, configurase como um conjunto de tecnologias e práticas que pode ser capaz de mobilizar esforços para a mudança necessária, de acordo com o preconizado pelo Movimento da Reforma Sanitária.

A promoção da saúde configura-se como estratégia de mudança nos modelos tecnoassistenciais, sinalizando a construção de outras possibilidades e a configuração de novos saberes e fazeres Que ampliem as alternativas de Qualidade de saúde e vida da população, de intervenção junto aos sujeitos e da compreensão do processo saúde-doença como produção social. Representa, pois, uma estratégia nos âmbitos político, assistencial, educacional e gerencial com um arcabouço conceitual e metodológico Que contribui para a transformação da lógica das ações de saúde.

Atuar na promoção da saúde configura-se como uma possibilidade de responder a demandas sociais e exige reflexões Que perpassam Quatro eixos fundamentais: a concepção de saúde, a gestão do processo de trabalho e educação, a formação dos profissionais de saúde a participação e o controle social. A conjugação dos elementos destes eixos deve direcionar as práticas em saúde, imprimindo a lógica do modelo tecnoassistencial em constante construção e reconstrução. Essa premissa resgata a imagem de um Quadrilátero para a formação em saúde ${ }^{(3)} \mathrm{em}$ Que ensino - gestão setorial - práticas de atenção - controle social propõem construir e organizar uma educação responsável por processos interativos e de ação na realidade para operar mudanças (desejo de futuro), mobilizar caminhos (negociar e pactuar processos), convocar protagonismos (pedagogia in acto) e detectar a paisagem interativa e móvel de indivíduos, coletivos e instituições como cenário de conhecimentos e invenções (cartografia permanente).

As mudanças nas práticas assistenciais direcionadas à construção da promoção da saúde são ainda incipientes e têm pequena visibilidade no cenário das práticas em saúde justificando, assim, a não percepção desta prática como estratégia para a transformação, tanto do ensino Quanto da gestão e do controle social.

No caso específico da enfermagem, a formação de profissionais graduados está sendo realizada no País por mais de 670 instituições sob a orientação das Leis de Diretrizes e Bases da Educação Brasileira - LDB ${ }^{(4)}$ e das Diretrizes Curriculares Nacionais - DCNpara os Cursos da Área da Saúde ${ }^{(5)}$. Portanto, configura-se como potencialmente capaz de influir na construção de novas práticas, entre elas aquelas relacionadas à promoção da saúde. Contudo, a literatura aponta Que, apesar de ter ocorrido grande avanço em sua formulação, a concepção de promoção da saúde não está devidamente incorporada aos projetos político-pedagógico dos cursos da área de saúde, nem às práticas educativas. As instituições formadoras têm perpetuado modelos essencialmente conservadores, consoantes com a ciência positivista, limitando-se a abordar a enfermidade, a cura e a atenção individual ${ }^{(6,7)}$.

Tomar os referenciais de promoção da saúde na ressignificação do ensino de enfermagem implica em transformação das práticas de ensino superando o modelo biologicista e a natureza setorial Que caracteriza a formação e a atuação dos profissionais de saúde. É preciso, também, Que o processo ensino-aprendizagem em enfermagem favoreça as práticas educacionais e de atenção à saúde Que potencializem o empoderamento dos sujeitos para atuarem na efetivação das mudanças sociais. É preciso propiciar um movimento dinâmico e de permanente ressignificação do conhecimento, de aquisição de habilidades e de atitudes Que os faça mais capazes para a vida e para o trabalho, assumindo-se, assim, a educação crítico-reflexiva.

\section{OB|ETIVOS}

O estudo apresenta resultados da pesquisa intitulada "Promoção da Saúde: estratégia política, assistencial, educacional e gerencial para a construção do modelo tecnoassistencial em saúde", desenvolvida por pesquisadoras do Núcleo de Estudos e Pesquisas sobre Ensino e Prática de Enfermagem (NUPEPE) da Escola de Enfermagem da Universidade Federal de Minas Gerais/Brasil.

O objetivo geral da pesøuisa foi analisar a promoção da saúde como estratégia para construção do modelo tecnoassistencial nos campos da política e da gestão do sistema de saúde, do ensino e da prática de equipes de saúde da família. Para cumprir este objetivo organizou-se a pesquisa em Quatro subprojetos: As políticas públicas de promoção da saúde - da gestão do sistema à articulação intersetorial; Concepções de promoção da saúde na eQuipe de saúde da família; Abordagem da promoção da saúde na formação do enfermeiro e Promoção da saúde em espaços sociais da vida cotidiana.

Especificamente, este trabalho trata da promoção da saúde no ensino de enfermagem, Que teve como objetivo geral analisar a abordagem da promoção da saúde na formação do enfermeiro. Foram definidos como objetivos específicos identificar as práticas 
de promoção da saúde desenvolvidas durante a formação; identificar os referenciais teórico-conceituais sobre promoção da saúde Que sustentam a formação do enfermeiro e identificar as relações existentes entre ensino-serviço-comunidade para viabilizar as práticas de promoção da saúde.

\section{MÉTODO}

O estudo caracteriza-se como de abordagem Qualitativa, orientado pela concepção teórico-filosófica da dialética ${ }^{(8)}$. As Questões éticas foram observadas em todas as etapas da pesQuisa, atendendo a Resolução 196/96 do Conselho Nacional de Ética, sendo a pesquisa aprovada pelo Comitê de Ética em Pesquisa da Universidade Federal de Minas Gerais.

Os cenários da pesquisa foram dois cursos de graduação em enfermagem do Estado de Minas Gerais/Brasil: uma instituição privada e uma instituição pública.

Em uma primeira fase foram entrevistadas as coordenadoras dos dois cursos, utilizando-se um roteiro semi-estruturado. No momento da entrevista, foi solicitado Que os coordenadores indicassem situações marcadoras de aprendizagem para a promoção da saúde, vivenciadas pelos sujeitos em formação naQuela instituição (marcadoras porQue representativas, ineuietantes ou exemplares). Para isso, uma das escolas incluiu a participação de outra docente, coordenadora de uma das práticas indicadas.

A segunda etapa da coleta de dados consistiu na verificação in loco das situações marcadoras indicadas, resultando em entrevistas com 2 docentes, 5 estudantes e 11 profissionais dos serviços de saúde Que recebem os estudantes de enfermagem.

Para a análise dos dados empíricos, utilizou-se a técnica de análise de discurso ${ }^{(8)}$. Foram realizadas leituras exaustivas dos discursos buscando apreender as idéias centrais, ou seja, estruturas de significados apresentadas nas falas dos participantes. O agrupamento das idéias centrais deu origem a uma rede de significados relacionados com as políticas e as ações pedagógicas Que favorecem a incorporação de concepções e práticas de promoção da saúde na formação do enfermeiro. A rede de significados foi analisada de acordo com os objetivos do estudo, originando 2 categorias empíricas: Projeto ético-político da enfermagem e a formação para a promoção da saúde; Articulação ensino-serviço e os desafios para uma nova forma de se fazer saúde.

A etapa final da análise consistiu na interpretação das categorias empíricas, estabelecendo diálogos com os autores consultados e a experiência e conhecimento das pesquisadoras, num verdadeiro movimento dialético, visando ao concreto pensado ${ }^{(8)}$ revelando as determinações e as especificidades Que se expressam nas concepções de promoção da saúde na formação do enfermeiro.

\section{RESULTADOS E DISCUSSÃO}

\section{Projeto Ético-Político da Enfermagem e a Formação para Promoção da Saúde}

As duas escolas cenários do estudo apresentam estruturas político-administrativas distintas, o Que as diferencia no seu funcionamento, na composição da força de trabalho docente, na concepção Que orienta o projeto político-pedagógico e nas práticas pedagógicas e de inserção nos cenários de aprendizagem. Contudo, ambas referenciam no seu projeto político-pedagógico as discussões nacionais sobre a formação do enfermeiro, em especial aQuelas referentes às Diretrizes Curriculares e aquelas relacionadas à abordagem da promoção da saúde enQuanto política pública.

As participantes do estudo Que exercem cargo de coordenação no curso apontam Que o movimento nacional de transformação do ensino em saúde, Que definiu as Diretrizes Curriculares Nacionais para os cursos da área, é um indutor importante para a incorporação da promoção da saúde como conteúdo e prática durante o processo de formação do enfermeiro. Além disso, reconhecem a importância de iniciativas governamentais de indução da formação crítica, reflexiva e criativa de profissionais de saúde, Que geram projetos e programas orientados por ações estratégicas, e Que contribuem para a construção de propostas Que buscam consolidar a cidadania, com a plena participação popular e controle social pelos diferentes setores da sociedade brasileira.

De fato, a implantação das DCN tem sido assumida como estratégia potente para redirecionar a formação dos profissionais de enfermagem, estabelecendo um marco estruturante na construção de um novo paradigma para a educação de enfermagem: a orientação da formação para impulsionar a efetivação dos princípios do SUS e das demandas e necessidades de saúde da população $^{(9)}$.

Entretanto, a proposição política ainda não foi capaz de se concretizar em mudanças nas práticas pedagógicas e os discursos dos participantes do estudo diferem consideravelmente ao expressarem as concepções Que orientam a formação do enfermeiro nas duas Escolas. Revela-se, em um dos discursos, a capacidade de formulação e reflexão crítica dos referenciais Que sustentam a promoção da saúde e sua relação no contexto de formação do enfermeiro. Expressões e conceitos sobre interdisciplinaridade, intersetorialidade, parcerias, relações com a sociedade civil organizada e grupos comunitários compõem o discurso, sugerindo o entendimento da importância destes temas para a discussão de um novo marco na referência de promoçãa da saúde. Neste discurso, ainda, é revelada a valorização dos desejos, sonhos e anseios das pessoas como elemento a ser considerado nas práticas Que têm como referência a promoção da saúde e, conseQüentemente, na formação do enfermeiro.

Os dois aspectos apontados por uma das participantes pode ser referenciado em Westphal ${ }^{(10)}$ Que admite haver dois enfoQues na potencialização das pessoas para a promoção da saúde. Um é o enfoque objetivo, voltado para a melhoria das condições de vida, com participação dos sujeitos na formulação de políticas públicas, integração social, capacidade de participar da vida social Que, implicitamente, remete à ampliação de poder e mobilização para o reconhecimento da saúde como direito social. O outro é um enfoque subjetivo, Que contempla os sentidos e significados da saúde e dos modos de viver; nesse enfoque, promover saúde com Qualidade de vida em suas múltiplas dimensões envolve a singularidade e a autonomia dos sujeitos. Considerar estes aspectos na formação do enfermeiro permite inferir Que o projeto ético-político da enfermagem deve produzir conhecimentos e uma prática social Que supere a reprodução de práticas hegemônicas e dominantes no campo da saúde, num verdadeiro processo de mudança da formação. 
Nos discursos das coordenadoras é revelada a compreensão de Que o processo de mudança na formação do enfermeiro é gradual e desafiante. Requer ações estratégicas no sentido de incorporar a promoção da saúde, como referencial no ensino e nas práticas sanitárias, e implicação de todos os atores envolvidos no processo ensino-aprendizagem.

As coordenadoras afirmam Que a formação do enfermeiro tem demandado esforços no sentido de construir modelos de ensino Que ofereçam ferramentas e desenvolvam as competências para atuação profissional, reconhecendo e contribuindo para a construção de novas práticas em saúde.

É importante destacar Que as concepções expressas pelas duas coordenadoras participantes do estudo podem não refletir as concepções do conjunto de professores e estudantes da Escolascenário. Contudo, ressalta-se Que a captação da compreensão das coordenadoras é importante pelo lugar Que elas ocupam na condução técnica, política e administrativa do Projeto Político Pedagógico e em especial do processo de ensino-aprendizagem.

\section{Articulação Ensino-Serviço e os Desafios para uma Nova Forma de se Fazer Saúde}

Os discursos das coordenadoras apontam Que no processo de formação há espaços para Que docentes e estudantes desenvolvam práticas de promoção da saúde. Em um dos discursos, é feita uma reflexão sobre o conceito de promoção apontando a imprecisão conceitual Que permeia, na formação e na atenção, as práticas denominadas de promoção da saúde. Ainda, revela o distanciamento entre a formulação conceitual e a concretude das práticas de promoção nos cenários de aprendizagem.

Realmente, há diferentes concepções sobre o termo promoção da saúde Que traduzem diferentes concepções acerca do processo saúde-doença $^{(1)}$. Admite-se Que as diversas conceituações disponíveis para a promoção da saúde são reunidas, segundo Buss $^{(12)}$, em dois grandes grupos: aquele em Que promoção da saúde consiste em um conjunto de atividades destinadas à transformação de comportamentos dos indivíduos, concentrando componentes educativos relacionados com riscos comportamentais passíveis de mudança (cita-se como exemplo as atividades físicas, dietas, hábito de fumar, direção perigosa no trânsito); outro grupo relaciona saúde a um amplo espectro de fatores relacionados com a Qualidade de vida, incluindo-se estilos de vida responsável, oportunidades de educação ao longo da vida, apoio social para famílias e indivíduos.

A verificação in loco das experiências indicadas como marcadoras da aprendizagem para promoção da saúde permitiu analisar o referencial de promoção da saúde Que orienta a formação e a relação ensino-serviço-comunidade subjacente à sustentabilidade deste conceito.

As práticas referidas pelos estudantes aproximam-se da abordagem sócio-política e de Qualidade de vida, envolvendo ações de empoderamento e ampliação da capacidade das comunidades nas decisões sobre a saúde, além do indispensável estabelecimento das parcerias intersetoriais na construção de políticas públicas incidem na saúde.

Em um dos cenários, os estudantes demonstraram reconhecer a importância de participação na gestão dos serviços, incluindo as relações com o gestor e com o controle social. Consideraram Que a prática mais importante de promoção da saúde desenvolvida no campo foi a capacitação dos Conselheiros Locais de Saúde. A possibilidade de análise destas relações pode ser incluída na perspectiva de fortalecimento da ação comunitária, um dos eixos de ação da promoção da saúde.

Em outro cenário, o trabalho de territorialização e mapeamento da área de abrangência da equipe de Saúde da Família, desenvolvido pelos estudantes, foi enfatizado como pertinente à promoção da saúde. Neste cenário, apesar do esforço dos estudantes, a relação com os usuários e com os outros serviços da comunidade revelouse bastante pontual.

Os estudantes das duas experiências indicadas como marcadoras de aprendizagem para promoção da saúde ressaltaram a importância do espaço do trabalho como lócus privilegiado de relações com os serviços de saúde e com a comunidade. Ressaltaram, ainda, Que esse espaço possibilita a expressão do espírito criativo, sendo favorável à aprendizagem.

Destaca-se como experiência importante, nos dois cenários, o Estágio Supervisionado em Saúde Coletiva ou Atenção Básica, desenvolvido nos dois semestres finais do curso. Ele é apontado, repetidamente, como espaço privilegiado para o desenvolvimento das práticas de promoção da saúde. Contudo, disciplinas do início do curso também são citadas como dispositivos potentes para disparar discussões sobre promoção da saúde, nos dois cursos, mesmo Que ainda estejam ocorrendo de forma pontual e por iniciativa dependente da concepção do professor.

A inserção dos alunos no campo, no caso do Estágio Supervisionado Que tem uma duração ampliada, permite a criação de vínculos com a população e desenvolvimento mais contínuo das atividades, contribuindo para o fortalecimento da autonomia dos estudantes. Nestes cenários de aprendizagem é possibilitado aos estudantes demonstrarem habilidades atributos para lidar com Questões éticas, políticas, administrativo-gerenciais, de resolução de conflitos, de trabalho em equipe, além das habilidades técnicas e cognitivas. Eles respondem a um contexto Que requer resposta imediata e com visão desse contexto ${ }^{(6)}$.

Nos discursos, as relações ensino-serviço-comunidade Que viabilizam as práticas de promoção da saúde foram expressas tendo como referência os avanços conseguidos nos dois cursos e os desafios a serem enfrentados para as mudanças estruturantes dos modelos de formação das duas instituições.

As parcerias são valorizadas pelos participantes como fator essencial para o alcance do perfil de egresso esperado pelas duas instituições-cenário, na perspectiva da promoção da saúde. As estratégias para concretização da integração entre a Universidade e os serviços são formais, como contratos e convênios, e aQuelas Que surgem das relações entre docentes, estudantes, profissionais de serviço e grupos da comunidade.

Os profissionais de serviço reconhecem a inserção do estudante como possibilidade de renovação de conhecimentos e, em conseqüência, das práticas, favorecendo o surgimento de propostas Que potencializam as ações de promoção da saúde. Estes profissionais descrevem a concepção de promoção da saúde associando-a ao conceito ampliado de saúde, tradicionalmente defendido pela Organização Mundial de Saúde. Embora assim o façam, indicam como práticas de promoção da saúde Que desenvolvem, aQuelas relacionadas à educação em saúde para 
mudança de comportamentos de risco.

Desta forma, corroboram a afirmação de Que é incipiente a incorporação da promoção da saúde nas práticas dos serviços e Que, muitas vezes, o termo é empregado de forma inadequada pelos profissionais de saúde, Que o confundem com práticas de prevenção e educação para a saúde ${ }^{(13)}$.

O confronto entre as concepções expressas pelos estudantes e pelos profissionais dos serviços de saúde leva-nos a Questionar a potência da presença dos estudantes na mudança das práticas em saúde, condição afirmada por um dos profissionais como principal motivação para a continuidade do estágio: "a presença dos estudantes provoca uma crise existencial nos serviços".

Infere-se Que essa contradição remete a dois determinantes interrelacionados:

$\left.1^{\circ}\right)$ A cristalização de práticas assistenciais em saúde Que reiteram um modelo no Qual as ações de saúde são realizadas apenas no sentido de agir sobre os efeitos do adoecer; ainda não foram incorporadas práticas a serem realizadas em espaços fora dos serviços de saúde, transcendendo a responsabilidade de proporcionar ações de recuperação da saúde com enfoQue curativo. Neste sentido, os desafios para imprimir uma nova lógica nas práticas de saúde, com enfoQue na promoção, exige re-significação das relações entre sujeitos, e dos sujeitos consigo mesmos, num movimento de construção de saberes e fazeres Que considera as subjetividades para a intervenção em saúde. As propostas devem incorporar o reconhecimento da liberdade na dinâmica de produção e realização dos indivíduos e das coletividades. $2^{\circ}$ ) A presença dos estudantes nos cenários é pontual e são poucos os mecanismos criados pelas instituições de ensino e de serviço para garantir a sustentabilidade das mudanças Que, potencialmente, poderiam ser provocadas nos serviços e no ensino. Assim, mesmo que potentes para imprimir uma nova lógica no modelo tecnoassistencial em saúde, é possível Que as rupturas e soluções de continuidade no processo enfraqueçam a capacidade das iniciativas disparadas.

Com essa análise, reconhece-se Que os princípios em Que se baseiam a formação do enfermeiro, nas duas Escolas, aproximamse dos campos de ação da promoção da saúde e implicam em transformação nas práticas de ensino, superando o modelo biologicista e a natureza setorial Que caracteriza a formação e a atuação dos profissionais de saúde. Passam a ser assumidos como um ideário do coletivo das Escolas. Entretanto, há carência de estratégias de integração docente/discente-serviço-comunidade como condição para a sustentabilidade do processo de mudança na formação do enfermeiro, mesmo ocorrendo a inserção do educando na realidade dos serviços e das comunidades.

Uma possibilidade de reversão deste Quadro a ser considerada é a construção de espaços de participação dos sujeitos dos três segmentos - universidade, serviço e comunidade - na elaboração de propostas de mudanças nos modelos de ensino e técnicoassistencial. Ou seja, o estabelecimento de instância tripartite para discussão, análise e deliberação sobre a situação atual e projeção de mudanças possíveis. Essa abordagem está ancorada na necessidade de construção de um sistema de saúde Que tem como pressuposto a concepção ampliada de saúde e Que se compromete com a regulação da formação dos profissionais para o setor.

A configuração dos cenários de aprendizagem traz consigo a temática da relação dos profissionais da universidade com os profissionais do serviço. Formar profissionais contextualizados à realidade dos serviços de saúde e Que atendam às demandas de "boas" práticas de promoção da saúde, exige Que universidade e serviços estejam juntos nas discussões do processo de formação. Suscita, portanto, a co-responsabilização do serviço pelo processo de formação.

\section{CONSIDERAÇÕES FINAIS}

Há um forte indicativo de Que a promoção da saúde é reconhecida como importante estratégia de renovação das práticas em saúde coletiva, re-configurando saberes e fazeres Que ampliem as alternativas de Qualidade de saúde e vida da população. Entretanto, as políticas e ações de promoção da saúde são ainda incipientes e tem demonstrado pouca capacidade de mudança nas práticas de atenção e de educação em enfermagem.

A realização desse estudo permitiu reafirmar a necessidades de mudanças no ensino de enfermagem Que superem modelos hegemônicos de educação, de gestão e de atenção à saúde. Ademais, pode contribuir para a construção/reconstrução de referenciais teóricos e metodológicos Que alicercem uma prática pedagógica e assistencial visando a promoção da saúde.

Enfatiza-se a necessidade de implementar, no ensino, o conceito ampliado do processo saúde-doença, adotando-se noções de promoção da saúde em contraposição à natureza setorial Que caracteriza a formação e a atuação dos profissionais. É preciso considerar os enfoques social, comunitário e político como determinantes das respostas efetivas em saúde. Portanto, para promover saúde é preciso construir políticas públicas intersetoriais voltadas para melhoria da Qualidade de vida, equidade na produção e consumo de ações e serviços de saúde, inclusão social e afirmação da cidadania.

No contexto da integração educação-saúde, a transformação das práticas reQuer uma atuação coletiva e consciente para superar os desafios e criar oportunidades para a melhoria da atenção à saúde e para a Qualificação do ensino. É imprescindível construir modelos Que primem pela autonomia dos sujeitos, pela afirmação dos princípios de cidadania e democracia, evidenciando o compromisso social com a melhoria do estado de saúde da população. Assim, é necessário pensar a promoção da saúde como um marco conceitual no redirecionamento do processo ensinoaprendizagem dos profissionais de saúde e de enfermagem.

\section{REFERÊNCIAS}

I. Fleury S. O dilema da Reforma Sanitária Brasileira. In: Berlinguer G, Fleury S, Campos GWS. Reforma Sanitária Itália e Brasil. São Paulo: HUCITEC; 1988. p. 195-207.
2. Feuerwerker LCM. Modelos tecnoassistenciais, gestão e organização do trabalho em saúde: nada é indiferente no processo de luta para a consolidação do SUS. Interface-Comunic Saúde Educ 2005; 9(18): 489-506. 
3. Ceccim RB; Feuerwerker LCM; O euadrilátero da formação para a área da saúde: ensino, gestão, atenção e controle social. Rev Saúde Coletiva 2004; 14(I): 41-65.

4. Ministério da Educação e Cultura (BR). Conselho Nacional de Educação. Lei 9394 de 20 de dezembro de 1996. Estabelece as Diretrizes e bases da Educação Nacional. Diário Oficial da União 1996 dez 23; 1: 27833-41.

5. Ministério da Educação e Cultura (BR). Conselho Nacional de Educação. Diretrizes Curriculares Nacionais do Curso de Graduação em Enfermagem. Diário Oficial da União 200 I nov 9; 1: 37.

6. Silva KL, Sena RR. A formação do enfermeiro: construindo a integralidade do cuidado. Rev Bras Enferm 2006;59(4): 48891.

7. Silva KL, Sena RR. Integralidade no cuidado em saúde: indicativos a partir da formação do enfermeiro. Rev Esc Enferm USP 2008; 42(1): 48-56.
8. Minayo MCS. O desafio do conhecimento: pesquisa Qualitativa em saúde. 10 $0^{\text {a. }}$ ed. São Paulo: Hucitec; 2007.

9. Silva KL, Sena RR. Nursing education: seeking critical-reflexive education and professional competencies. Rev Latino-am Enfermagem 2006; I 4(5): 755-61.

10. Westphal MF. Promoção da saúde e prevenção de doenças. In: Campos GWS. Tratado de Saúde Coletiva. São Paulo: Hucitec; 2006. p. 635-68.

11. Czeresnia D. O conceito de saúde e a diferença entre prevenção e promoção. In: Czeresnia D, Freitas CM, organizadores Promoção da saúde: conceitos, reflexões, tendências. Rio de Ianeiro: FIOCRUZ; 2003. p. 39-54.

12. Buss PM. Promoção da saúde e Qualidade de vida. Ciência Saúde Coletiva 2000; 5(1): 163-77.

13. Souza EM, Grundy E. Promoção da saúde, epidemiologia social e capital social: inter-relações e perspectivas para a saúde pública. Cad Saúde Pública 2004; 20(5): 1354-60. 